\title{
Symptomatic remission in psychosis and real-life functioning
}

\author{
M. Oorschot, T. Lataster, V. Thewissen, M. Lardinois, J. van Os, P. A. E. G. Delespaul
}

and I. Myin-Germeys

\section{Background}

In 2005 Andreasen proposed criteria for remission in schizophrenia. It is unclear whether these criteria reflect symptom reduction and improved social functioning in daily life.

\section{Aims}

To investigate whether criteria for symptomatic remission reflect symptom reduction and improved functioning in real life, comparing patients meeting remission criteria, patients not meeting these criteria and healthy controls.

\section{Method}

The Experience Sampling Method (ESM), a structured diary technique, was used to explore real-life symptoms and functioning in 177 patients with (remitted and non-remitted) schizophrenia spectrum disorders and 148 controls.

\section{Results}

Of 177 patients, 70 met criteria for symptomatic remission. These patients reported significantly fewer positive and negative symptoms and better mood states compared with patients not in remission. Furthermore, patients in remission spent more time in goal-directed activities and had less preference for being alone when they were with others However, the patient groups did not differ on time spent in social company and doing nothing, and both the remission and non-remission groups had lower scores on functional outcome measures compared with the control group.

\section{Conclusions}

The study provides an ecological validation for the symptomatic remission criteria, showing that patients who met the criteria reported fewer positive symptoms, better mood states and partial recovery of reward experience compared with those not in remission. However, remission status was not related to functional recovery, suggesting that the current focus on symptomatic remission may reflect an overly restricted goal.

\section{Declaration of interest}

None.
The consensus definition of remission in schizophrenia defines remission as:

a state in which patients have experienced an improvement in core signs and symptoms to the extent that any remaining symptoms are of such low intensity that they no longer interfere significantly with behavior and are below the threshold typically utilized in justifying an initial diagnosis of schizophrenia.

The operationalisation of this definition consists of two elements: the absence or low intensity of eight core symptoms of schizophrenia (severity criterion) and the maintenance of this state for a minimum of 6 months (time criterion). The consensus definition is aimed at facilitating research on the course of illness, at improving the comparability of studies and at helping clinicians and patients focus on positively formulated treatment outcomes. ${ }^{1,2}$ The definition has been used in several studies of schizophrenia, exploring cognitive ability and antipsychotic medication, ${ }^{3,4}$ and is validated by several studies using functiona outcome, symptom severity, need for care and/or quality of life as outcome measures. ${ }^{5-9}$ However, these outcome measures are mostly clinician-rated and standardised, whereas real-life validation using self-report measures of actual functioning (e.g. time spent with others and in goal-directed activities) and real-life experiences is lacking. We therefore aimed to validate the remission criterion using a momentary assessment strategy investigating symptom level and functioning in patients with schizophrenia spectrum disorders. We investigated positive symptoms, negative symptoms and functional outcome in the flow of daily life in patients meeting the severity criterion of the symptomatic remission definition, patients not meeting that criterion and a healthy comparison group, the last allowing a comparison with a 'normal' level of functioning.

\section{Method}

The sample consisted of 191 patients diagnosed with schizophrenia spectrum disorders and a control group of 168 healthy individuals. Data for this study were pooled from three previous Experience Sampling Method (ESM) studies. $^{10-12}$ Inclusion criteria for all studies were age 18-65 years and sufficient command of the Dutch language to understand and complete the questionnaires. Exclusion criteria were brain disease and history of head injury with loss of consciousness. Control participants were excluded if presenting with a lifetime history of psychotic or affective disorder or a family history of psychotic disorder. In all three studies, patients were recruited from mental health facilities in the south of The Netherlands and in Flanders, Belgium. A full description of this procedure is given in the earlier studies of the three study samples. ${ }^{11-13}$ Interview data and clinical record data were used to complete the Operational Criteria Checklist for Psychotic Illness yielding DSM-III-R and DSM-IV diagnoses in two of the study samples, ${ }^{11,12}$ or the Comprehensive Assessment of Symptoms and History, ${ }^{14}$ yielding DSM-IV diagnoses in one study sample. ${ }^{13}$ The ESM questionnaires were set up identically in terms of mood, symptoms and context to enable pooling of the data. Written informed consent, conforming to local ethics committee guidelines, was obtained from all participants. Participants were compensated with a voucher of $€ 25$ (or equivalent).

\section{Experience Sampling Method}

The ESM, a structured self-assessment technique, was used to collect data in the natural flow of daily life. ${ }^{15,16}$ Participants received a 
pre-programmed digital wristwatch and ten self-assessment forms collated in a booklet for each day. Ten times a day on six consecutive days the watch emitted a signal at unpredictable moments between $07.30 \mathrm{~h}$ and $22.30 \mathrm{~h}$. After each 'beep' participants were to fill out one of the forms, rating emotional experience, symptoms and context on seven-point Likert scales and answering open-ended questions. The ESM procedure was explained in a briefing session, in which all participants completed a practice form, were instructed to complete their reports immediately after the beep and to register the time at which they completed the questionnaire. During the sampling period, research staff contacted participants to assess whether they were complying with the instructions. In a debriefing session participants were interviewed to be sure that they had complied with the instruction. Reports were assumed to be valid when participants responded to the beep within $15 \mathrm{~min}$, and their data were only included in the analyses when they provided valid responses for at least a third of the emitted beeps. ${ }^{17}$ Previous studies have demonstrated the feasibility, validity and reliability of ESM in general and patient populations. ${ }^{16,18}$ The following variables were derived from the ESM questionnaires.

\section{Positive symptom assessment}

Hallucinations were measured using the items 'I hear voices' and 'I see phenomena'. Delusional intensity was measured using three items ('I'm suspicious', 'I can't get rid of my thoughts' and 'I fear losing control'; Cronbach's $\alpha=0.66$ ). The validity of the delusion and hallucination items had been previously demonstrated. ${ }^{19,20}$ All items were rated on a Likert scale ranging from 1 (not at all) to 7 (very).

\section{Negative symptom assessment}

\section{Flattened emotional experience}

Emotional experience was assessed with eight mood adjectives (e.g. 'I feel anxious') rated on seven-point Likert scales. The items 'insecure', 'lonely', 'anxious', 'sad' and 'guilty' constituted negative affect (Cronbach's $\alpha=0.83$ ). The mean score of the items 'cheerful', 'relaxed' and 'satisfied' constituted positive affect $(\alpha=0.84)$.

\section{Anhedonia}

Anhedonia was defined as lack of emotional reward after pleasant events, with emotional reward conceptualised as the change in positive affect after pleasant events compared with that after neutral events. ${ }^{21}$ In order to assess anhedonia, participants were asked to report the most important event that had happened between the current and the previous beep. Subsequently, the participant rated this event on a seven-point bipolar scale $(-3$ very unpleasant, 0 neutral, 3 very pleasant), providing a subjective measure of event pleasantness. Observations including events appraised as slightly pleasant (1), pleasant (2), very pleasant (3) and neutral (0) were included in the analyses. The neutral events were set as the reference category. The effect of subjective (positive) event pleasantness on positive affect was calculated, with lower positive affect reactions indicating more anhedonia.

\section{Functional outcome}

Social functioning

Real-life social functioning was conceptualised using self-report information regarding participants' social context and the appraisal thereof. Participants were asked to report whether they were alone; if not, they had to report how much they would prefer to be alone ('I'd rather be alone') and to indicate the actual level of interaction using the item 'We are interacting', both rated on a seven-point Likert scale ( 1 not at all, 7 very). We investigated time spent alone, level of interaction and preference for being alone while with others. We furthermore measured the level of social anhedonia, which was defined as lack of emotional reward from being in the company of others, with emotional reward conceptualised as the change in positive affect when with others compared with being alone. ${ }^{22}$ We also investigated the change in negative affect when with others compared with being alone in order to examine the possible negative effects of social company on emotional experience.

\section{Activity level}

In order to assess activity level, participants were asked to report what they were doing. These activities were coded and divided in 'doing nothing' $v$. 'doing something', and 'goal-directed activities' (e.g. household chores, study/work) $v$. 'not goal-directed activities' (e.g. watching television, taking a walk). Subjective activity level was measured using the item 'I'm active' rated on a seven-point Likert scale.

\section{Remission}

Interview-based information on symptom severity was assessed with the Positive and Negative Syndrome Scale (PANSS), ${ }^{23}$ a semi-structured interview rating positive (7 items), negative (7 items) and general (16 items) symptoms. Each item was scored on a scale ranging from 1 (absent) to 7 (extreme). Assessment was done by a trained research assistant within a week after the sampling period. Remission status was defined as a score of 3 or below on the following PANSS items: delusions, unusual thought content, hallucinatory behaviour, conceptual disorganisation, mannerism/posturing, blunted affect, passive/apathetic social withdrawal and lack of spontaneity and flow of conversation. Participants were divided in three groups ( 0 control group, 1 remission group, 2 non-remission group).

\section{Statistical analysis}

Multilevel linear modelling techniques were used to examine the associations between remission status and outcome measures. Multilevel or hierarchical linear modelling techniques are a variant of the more often used unilevel linear regression analyses and are ideally suited for the analysis of ESM data consisting of multiple observations in one person, creating two levels of analysis (ESM beep level and participant level). Data were analysed with the XTREG module in Stata version 10.0 on Windows. Effect sizes from predictors in the multilevel model were expressed as $B$, representing the fixed regression coefficient. In all analyses we investigated the effect of group on the dependent variable. Gender and age were included a priori as confounders in all regression models.

Analyses of positive symptoms were conducted with hallucinatory or delusional intensity as dependent variable and group as independent variable. Flattened emotional experience was analysed with level of positive or negative affect as dependent variable and group as independent variable. Anhedonia was investigated first with the number of positive events as dependent variable and group as independent variable, and second in a random regression model with positive affect as dependent variable and event pleasantness, group and the interaction between these as independent variables. We included negative affect intensity and number of observations as covariates in the analyses. From these models, effect sizes of event pleasantness, stratified by group, were calculated by applying and testing the 
appropriate linear combinations using the Stata LINCOM command. Main effects and interactions were assessed by Wald tests. Social functioning was analysed with percentage of moments alone, level of interaction and preference for being alone as dependent variables and group as independent variable. Differences in the effect of being in the company of others on emotional experience were investigated fitting multilevel random regression models with positive or negative affect as dependent variable and group, social context ( 0 not alone, 1 alone) and their interaction as independent variables. Activity level was analysed with percentage of moments spent in goal-directed activities and subjective activity level as dependent variables and group as independent variable.

\section{Results}

Of the recruited participants, 3 patients (having mania with psychotic features as their main diagnosis) and 17 persons from the control group (with a lifetime history of depression) were excluded from the analyses. Furthermore, 9 patients (3 in remission) and 3 control group members were excluded because of insufficient number of valid ESM observations (fewer than 20) and 2 patients were excluded because of missing data on the PANSS. The final sample therefore comprised 177 patients (70 in remission) and 148 controls. Additional information regarding sociodemographic characteristics and ESM reports is summarised in Table 1. Mean scores on the dependent and independent variables are summarised in Table 2.

\section{Positive symptoms}

Patients who were not in remission reported significantly higher levels of hallucinatory and delusional intensity compared with both the remission group and the control group (Table 3). Patients in remission scored more highly on delusional intensity compared with the control group (Table 3 ).

\section{Negative symptoms}

Both patient groups reported significantly lower positive affect and higher negative affect compared with controls. However, the

\begin{tabular}{l} 
Table 1 Demographic and clinical characteristics \\
\cline { 2 - 3 }
\end{tabular}

\begin{tabular}{|c|c|c|c|}
\hline & $\begin{array}{l}\text { Non-remission group } \\
\qquad(n=107)\end{array}$ & $\begin{array}{l}\text { Remission group } \\
\qquad(n=70)\end{array}$ & $\begin{array}{l}\text { Control group } \\
\quad(n=148)\end{array}$ \\
\hline \multicolumn{4}{|l|}{ Positive symptoms, mean (s.d.) } \\
\hline Auditory hallucinations & $1.95(1.74)$ & $1.02(0.23)$ & $1.01(0.15)$ \\
\hline Visual hallucinations & $1.44(1.27)$ & $1.02(0.24)$ & $1.01(0.11)$ \\
\hline Delusional intensity & $2.00(1.24)$ & $1.30(0.63)$ & $1.12(0.38)$ \\
\hline \multicolumn{4}{|l|}{ Negative symptoms, mean (s.d.) } \\
\hline Negative affect & $2.11(1.16)$ & $1.54(0.80)$ & $1.24(0.48)$ \\
\hline Positive affect & $4.24(1.38)$ & $4.82(1.24)$ & $5.32(1.02)$ \\
\hline Number of positive events, mean & 32 & 35 & 40 \\
\hline \multicolumn{4}{|l|}{ Functioning } \\
\hline Percentage of time spent alone, mean & 43 & 42 & 35 \\
\hline Level of interaction, mean (s.d.) & $3.76(2.25)$ & $4.13(2.25)$ & $4.14(2.36)$ \\
\hline Preference for being alone, mean (s.d.) & $2.40(1.96)$ & $1.81(1.53)$ & $1.56(1.23)$ \\
\hline Percentage of time spent doing nothing, mean & 10 & 11 & 4 \\
\hline Percentage of time spent in goal-directed activities, mean & 27 & 34 & 57 \\
\hline Activity level, mean (s.d.) & $3.70(2.01)$ & $3.38(1.99)$ & $3.76(1.99)$ \\
\hline
\end{tabular}




\begin{tabular}{|c|c|c|c|c|c|c|c|}
\hline & \multicolumn{3}{|c|}{ Non-remission group } & \multicolumn{3}{|c|}{ Remission group } & \multirow[b]{2}{*}{ Non-remission $v$. remission } \\
\hline & $B^{\mathrm{a}}$ & $95 \% \mathrm{Cl}$ & $P$ & $B^{\mathrm{a}}$ & $95 \% \mathrm{Cl}$ & $P$ & \\
\hline \multicolumn{8}{|l|}{ Positive symptoms } \\
\hline Auditory hallucinations & 0.98 & 0.75 to 1.20 & $<0.001$ & 0.04 & -0.21 to 0.29 & 0.76 & $\chi^{2}(1)=50.33, P<0.001$ \\
\hline Visual hallucinations & 0.44 & 0.28 to 0.61 & $<0.001$ & -0.004 & -0.19 to 0.18 & 0.96 & $\chi^{2}(1)=21.81, P<0.001$ \\
\hline Delusional intensity & 0.87 & 0.71 to 1.04 & $<0.001$ & 0.22 & 0.04 to 0.41 & 0.02 & $\chi^{2}(1)=43.99, P<0.001$ \\
\hline \multicolumn{8}{|l|}{ Negative symptoms } \\
\hline Negative affect & 0.87 & 0.72 to 1.04 & $<0.001$ & 0.31 & 0.12 to 0.49 & 0.001 & $\chi^{2}(1)=33.96, P<0.001$ \\
\hline Positive affect & -1.01 & -1.23 to -0.78 & $<0.001$ & -0.43 & -0.69 to -0.18 & 0.001 & $\chi^{2}(1)=18.46, P<0.001$ \\
\hline Number of positive events & -8.27 & -10.97 to -5.57 & $<0.001$ & -4.17 & -7.21 to -1.14 & 0.007 & $F(1,317)=6.64, P=0.01$ \\
\hline \multicolumn{8}{|l|}{ Functioning } \\
\hline Time spent alone & 0.06 & 0.006 to 0.11 & 0.03 & 0.06 & -0.001 to 0.12 & 0.05 & $F(1,316)=0.00, P=0.99$ \\
\hline Level of interaction & -0.24 & -0.63 to 0.15 & 0.15 & 0.05 & -0.32 to 0.42 & 0.79 & $\chi^{2}(1)=1.60, P=0.21$ \\
\hline Preference for being alone & 0.71 & 0.45 to 0.97 & $<0.001$ & 0.26 & -0.04 to 0.55 & 0.09 & $\chi^{2}(1)=8.59, P=0.003$ \\
\hline Time spent doing nothing & 0.08 & 0.06 to 0.10 & $<0.001$ & 0.07 & 0.05 to 0.10 & $<0.001$ & $F(1,316)=0.12, P=0.73$ \\
\hline Time spent in goal-directed activities & -0.29 & -0.33 to -0.25 & $<0.001$ & -0.22 & -0.27 to -0.18 & $<0.001$ & $F(1,316)=7.72, P=0.006$ \\
\hline Activity level & 0.06 & -0.23 to 0.35 & 0.67 & -0.08 & -0.41 to 0.24 & 0.62 & $\chi^{2}(1)=0.72, P=0.39$ \\
\hline
\end{tabular}

a. Regression coefficient indicates the difference in symptoms and functioning in the patient groups compared with the control group. Gender and age are included as confounders in the model.

two patient groups differed significantly from each other, with those in remission reporting higher positive affect and lower negative affect compared with the non-remission group. Both patient groups also reported significantly fewer positive events compared with controls, with patients in remission reporting more positive events than the non-remission group (Table 3). A significant interaction effect between event pleasantness and group in the model of positive affect was found $\left(\chi^{2}(2)=8.30, P=0.02\right)$, indicating that the groups differed in the level of positive affect reported after pleasant events. Analyses stratified by group revealed that event pleasantness and positive affect are positively associated in all groups (control group $B=0.09$, 95\% CI $0.07-$ $0.11, P<0.001$; remission group $B=0.14,95 \%$ CI $0.11-0.18$, $P<0.001$; non-remission group $B=0.10,95 \%$ CI $0.07-0.12$, $P<0.001)$. Patients in the remission group, however, displayed a larger increase in positive affect after pleasant events compared with the non-remission group $\left(\chi^{2}(1)=4.57, P=0.03\right)$ and controls $\left(\chi^{2}(1)=8.15, P=0.004\right)$, whereas the non-remission and control groups did not differ from each other $\left(\chi^{2}(1)=0.26, P=0.61\right)$.

\section{Functioning}

\section{Social functioning}

Patients spent significantly more time alone than control group participants, but the two patient groups did not significantly differ in the amount of time they spent with others (Table 3 ). In the company of others, the groups did not significantly differ in the intensity of interaction they reported. Patients, however, displayed a greater preference for being alone when with others compared with controls, and this preference for being alone was lower in the remission group than the non-remission group (Table 3). No significant interaction effect between social company and group was found in the model of positive affect $\left(\chi^{2}(2)=0.92\right.$, $P=0.63)$. Positive affect was decreased in all participants when they were alone $(B=-0.13,95 \% \mathrm{CI}-0.16$ to $-0.10, P<0.001)$. However, a significant interaction effect between social company and group was found in the model of negative affect $\left(\chi^{2}(2)=11.92, P=0.003\right)$. Analyses stratified by group revealed that being alone was associated with significant increased negative affect in patients (remission group $B=0.08,95 \%$ CI 0.03 to 0.12 , $P=0.001$; non-remission group $B=0.11,95 \%$ CI 0.07 to 0.14 , $P<0.001$; remission $v$. non-remission groups $\chi^{2}(1)=0.90$,
$P=0.34)$ but not in the control group $(B=0.02,95 \% \mathrm{CI}$ -0.004 to $0.05, P=0.09$ ).

\section{Activity level}

No difference was found in the subjectively experienced activity level between the three groups. However, both patient groups spent more time doing nothing and were less often involved in goal-directed activities compared with the control group. The remission group patients were more involved in goal-directed activities than patients not in remission (Table 3 ).

\section{Discussion}

Our findings provide ecological validation for the symptomatic remission criteria, showing that patients who met the severity criterion reported fewer positive symptoms, better mood states and increased hedonic capacity compared with patients with non-remitted disorder. Remission status, however, was not related to clear improvements in real-life functioning, since both remission and non-remission groups scored lower on our measures of functional outcome compared with controls. Subtle differences between the patient groups in this domain were present, however: patients in remission were found to spend more time performing goal-directed activities and showed less preference for being alone when with others compared with the non-remission group.

\section{Positive and negative symptoms}

Patients in remission showed reduced levels of hallucinations and delusional intensity compared with patients who were not. This is not surprising, since positive symptoms are relatively clearly defined and are directly reflected in the ESM measures of positive symptoms. The higher delusional intensity in patients in remission compared with the control group is in line with the remission criteria, which do not require symptoms to be completely absent. In contrast to the positive symptoms, negative symptom consensus items cannot be directly measured using ESM. Correct operationalisation of negative symptoms is further complicated by the current debate on traditional definitions and 
the nature of such symptoms. ${ }^{24}$ However, the study shows increased intensity of negative affect, which is indicative of the absence of flattened affective experience. This is in line with a growing number of studies showing the absence of flattened emotional experience in patients with schizophrenia, despite the fact that flattened emotional expression is present. ${ }^{25}$ Anhedonia is not measured using the PANSS and thus is not included in the consensus definition of remission. Nevertheless, our data reveal that hedonic capacity is improved in patients in remission. Hedonic capacity, or emotional reward experience, is proposed to be a critical factor underlying deficits in motivation and reallife functioning in schizophrenia, although studies of hedonic capacity in schizophrenia have inconsistent results. ${ }^{26}$

The increase in reward experience should be investigated in more depth since it may be one of the crucial mechanisms involved in recovery. Studies of reward experience and illness course or treatment effects in schizophrenia are surprisingly scarce. Several ESM studies in depression, however, showed changes in positive emotions and reward experience to be a predictor of treatment response to antidepressants. ${ }^{27,28}$ In our study, patients in remission reported both more pleasant events in their daily life and increased emotional reward from these pleasant events. These concepts are likely to be interrelated, with increased reward experience predicting increased motivation to search for positive experiences and the combination of more pleasant experiences and more emotional reward from such experiences working synergistically in their effect on mood. Increased reward experience or hedonic capacity might therefore be one of the keys to recovery after a psychotic episode and should be further investigated.

\section{Functional outcome}

The study shows that symptomatic remission is not the same as functional recovery; although patients in remission reported reduced symptom levels, real-life functioning did not clearly improve and this group still scored worse than controls on most measures of such functioning. This is in line with other studies, ${ }^{29}$ and with the focus of the remission criteria consensus group which was on symptomatic rather than functional remission. However, the consensus group decided on these specific symptom severity thresholds since they were assumed not to interfere significantly with day-to-day functioning, whereas our results suggest that functioning in patients with remitted disorder is still impaired. These impairments are not necessarily related to increased delusionality levels, but may be related to lower mood or other non-measured factors such as cognitive dysfunction. ${ }^{30}$ Moreover, many patients have never achieved certain social, educational or vocational milestones and functional impairments in the remission group could therefore just be a continuation of poor premorbid functioning. ${ }^{31}$

\section{Measuring real-world functioning}

In order to better understand the process of functional recovery, however, sophisticated measures of everyday functioning in schizophrenia are necessary. It is now generally accepted that symptomatic remission is too restricted a goal and that treatment should aim at functional remission. ${ }^{1,7}$ A widely accepted definition of functional remission is still lacking. Generally, functional remission is considered a multidimensional concept which is broader than symptomatic remission and implies good social and occupational functioning. ${ }^{31,32}$ A review of measures of social functioning in schizophrenia indicated that the most frequently used scales were the Global Assessment of Functioning Scale, the
Global Assessment Scale and the Social Functioning Scale. ${ }^{33}$ The first two measures, however, are both single-item, clinician-rated assessments of functioning, and the third is a self-report measure with a 3-month reference period. The ESM may be a useful addition to these measures of real-world functioning, since it is a self-report measure which is sensitive to small changes in behaviour, focuses on functioning in the realm of daily life and allows investigation of subjective appraisal of activities. In contrast to the traditional scales measuring real-life functioning, ESM allows investigation not only of functional outcome but also of the underlying processes and thus provides useful information for treatment and rehabilitation. Computerised ESM using mobile telephones, personal digital assistants or dedicated devices is a rapidly growing field in psychiatry research, ${ }^{34,35}$ and makes implementation of momentary assessment easier to achieve in clinical practice, since it minimises the effort and time required from both patient (filling in answers to the questions) and clinician (transcribing and analysing the data).

\section{Methodological issues}

Several methodological issues should be taken into account. First, as in other studies, ${ }^{5,7,8,36}$ we had no information on the 6-month time criterion. A longer period of symptomatic remission could result in improvement in functional outcome. However, we investigated functional outcome in a subset of 25 patients with remitted disorder for whom we did have time criterion data (16 fulfilling the 6-month criterion). These pilot results (not shown) indicated that functional recovery remains equally if not more problematic over time in patients fulfilling both the symptomatic and time criteria. Second, compliance with the research protocol is a crucial element of this research method. Some authors have cast doubt on compliance in paper-and-pencil ESM studies and preferred the use of electronic devices. ${ }^{37}$ However, two studies in which paper-and-pencil diary and electronic diary data were collected using comparable procedures suggested good compliance rates with the time protocol and demonstrated that both methods yielded data comparable in terms of both psychometric features and research findings. ${ }^{38,39}$ Third, in line with other studies by our group, ${ }^{10-12,19}$ participants who made a valid response to at least a third of the beeps were included in the analyses. Although this criterion might seem liberal compared with criteria used in studies applying more regular methods such as questionnaires and interviews, the absence of data is inherent to the ESM in which we study the daily life of participants while encouraging them not to adapt their activities to the research method. Moreover, the mean number of valid beeps in this study was two-thirds of the total number of beeps. We do not have evidence of systematic differences between groups on missed beeps, except for patients missing the first beep of the day more often than controls, which might be related to differences in sleeping pattern. ${ }^{17}$ Fourth, we used a scientific definition of remission and functioning, whereas patients have developed an experience-based approach to remission and recovery. Patientbased definitions of recovery generally refer to a unique and personal process in which people are able to participate fully in their communities and live a fulfilling and productive life despite a disability. ${ }^{40}$ Fifth, we included a control group of healthy individuals. One could argue that this group is not feasible as a control since its members also differ on all demographic variables; however, we chose to include this group to reflect a 'normal' level of functioning in society. Sixth, we pooled data from three different studies, which might induce systematic differences within the data-set; however, we feel that it is justified and necessary to pool data to increase power and find subtle effects, as is common 
practice in genetic studies. Furthermore, the diary structure was similar in all three studies and none of the data had been used previously to study remission or a related concept.

M. Oorschot, MSC, T. Lataster, PhD, Department of Psychiatry and Neuropsychology, Maastricht University, The Netherlands; V. Thewissen, PhD, Department of Psychiatry and Neuropsychology, Maastricht University, and Faculty of Psychology, Open University of the Netherlands, Heerlen; M. Lardinois, PhD, Department of Psychiatry and Neuropsychology, Maastricht University;

J. van Os, PhD, Department of Psychiatry and Neuropsychology, Maastricht University, The Netherlands, and Department of Psychosis Studies, Institute of Psychiatry, King's College London, UK; P. A. E. G. Delespaul, PhD, Department of Psychiatry and Neuropsychology, Maastricht University, The Netherlands; I. Myin-Germeys, PhD, Department of Psychiatry and Neuropsychology, Maastricht University, The Netherlands, and School of Psychological Sciences, University of Manchester, UK

Correspondence: Dr Inez Myin-Germeys, Department of Psychiatry and Neuropsychology, Maastricht University, PO Box 616 (VIJV), 6200 MD Maastricht, The Netherlands. Email: i.germeys@maastrichtuniversity.nl

First received 10 Oct 2011, final revision 12 Feb 2012, accepted 3 Apr 2012

\section{Funding}

I.M.-G. was supported by a 2006 NARSAD Young Investigator Award and by a Dutch Medical Research Council (Vidi) grant.

\section{References}

1 Andreasen N, Carpenter WT, Kane JM, Lasser RA, Marder SR, Weinberger DR. Remission in schizophrenia: proposed criteria and rationale for consensus. Am J Psychiatry 2005; 162: 441-9.

2 van Os J, Burns T, Cavallaro R, Leucht S, Peuskens J, Helldin L, et al. Standardized remission criteria in schizophrenia. Acta Psychiatr Scand 2006; 113: $91-5$.

3 Helldin L, Kane JM, Karilampi U, Norlander T, Archer T. Remission and cognitive ability in a cohort of patients with schizophrenia. J Psychiatr Res 2006; 40: 738-45.

4 Sethuraman G, Taylor CC, Enerson M, Dunayevich E. A retrospective comparison of cumulative time spent in remission during treatment with olanzapine or risperidone among patients with schizophrenia. Schizophr Res 2005: 79: 337-40.

5 Boden R, Sundstrom J, Lindstrom E, Lindstrom L. Association between symptomatic remission and functional outcome in first-episode schizophrenia. Schizophr Res 2009; 107: 232-7.

6 De Hert M, van Winkel R, Wampers M, Kane J, van Os J, Peuskens J. Remission criteria for schizophrenia: evaluation in a large naturalistic cohort Schizophr Res 2007; 92: 68-73.

7 Meesters PD, Comijs HC, de Haan L, Smit JH, Eikelenboom P, Beekman AT, et al. Symptomatic remission and associated factors in a catchment area based population of older patients with schizophrenia. Schizophr Res 2011 126: $237-44$.

8 van Os J, Drukker $\mathrm{M}$, à Campo J, Meijer J, Bak M, Delespaul P. Validation of remission criteria for schizophrenia. Am J Psychiatry 2006; 163: 2000-2.

9 Wunderink L, Nienhuis FJ, Sytema S, Wiersma D. Predictive validity of proposed remission criteria in first-episode schizophrenic patients responding to antipsychotics. Schizophr Bull 2007; 33: 792-6.

10 Lataster T, Collip D, Lardinois M, van Os J, Myin-Germeys I. Evidence for a familial correlation between increased reactivity to stress and positive psychotic symptoms. Acta Psychiatr Scand 2010; 122: 395-404.

11 Myin-Germeys I, Delespaul $P$, deVries MW. Schizophrenia patients are more emotionally active than is assumed based on their behavior. Schizophr Bull 2000; 26: 847-54

12 Thewissen V, Bentall RP, Lecomte T, van Os J, Myin-Germeys I. Fluctuation in self-esteem and paranoia in the context of daily life. J Abnorm Psychol 2008; 117: 143-53.

13 Lataster T, Collip D, Lardinois M, van Os J, Myin-Germeys I. Evidence for a familial correlation between Increased reactivity to stress and positive psychotic symptoms. Acta Psychiatr Scand 2010; 122: 395-404.

14 Andreasen N, Flaum M, Arndt S. The Comprehensive Assessment of Symptoms and History (CASH). An instrument for assessing diagnosis and psychopathology. Arch Gen Psychiatry 1992; 49: 615-23.
15 Csikszentmihalyi M, Larson R. Being Adolescents: Conflict and Growth in the Teenage Years. Basic Books, 1984.

16 Myin-Germeys I, Oorschot M, Collip D, Lataster J, Delespaul P, van Os J. Experience sampling research in psychopathology: opening the black box of daily life. Psychol Med 2009; 39: 1533-47.

17 Delespaul $\mathrm{P}$ (ed). Assessing Schizophrenia in Daily Life. The Experience Sampling Method. Maastricht University Press, 1995.

18 Oorschot M, Kwapil T, Delespaul P, Myin-Germeys I. Momentary assessment research in psychosis. Psychol Assess 2009; 21: 498-505.

19 Myin-Germeys I, Delespaul P, van Os J. Behavioural sensitization to daily life stress in psychosis. Psychol Med 2005; 35: 733-41.

20 Delespaul $\mathrm{P}$, deVries $\mathrm{M}$, van Os J. Determinants of occurrence and recovery from hallucinations in daily life. Soc Psychiatry Psychiatr Epidemiol 2002; 37: 97-104.

21 Wichers M, Aguilera M, Kenis G, Krabbendam L, Myin-Germeys I, Jacobs N, et al. The catechol-O-methyl transferase Val158Met polymorphism and experience of reward in the flow of daily life. Neuropsychopharmacology 2008; 33: 3030-6.

22 Kwapil TR, Silvia PJ, Myin-Germeys I, Anderson AJ, Coates SA, Brown LH. The social world of the socially anhedonic: exploring the daily ecology of asociality J Res Personality 2009; 43: 103-6.

23 Kay SR, Fiszbein A, Opler LA. The positive and negative syndrome scale (PANSS) for schizophrenia. Schizophr Bull 1987; 13: 261-76.

24 Foussias G, Remington G. Negative symptoms in schizophrenia: avolition and Occam's razor. Schizophr Bull 2010; 36: 359-69.

25 Kring AM, Moran EK. Emotional response deficits in schizophrenia: insights from affective science. Schizophr Bull 2008; 34: 819-34.

26 Horan WP, Kring AM, Blanchard JJ. Anhedonia in schizophrenia: a review of assessment strategies. Schizophr Bull 2006; 32: 259-73.

27 Geschwind N, Nicolson NA, Peeters F, van Os J, Barge-Schaapveld D, Wichers M. Early improvement in positive rather than negative emotion predicts remission from depression after pharmacotherapy. Eur Neuropsychopharmacol 21: 241-7.

28 Wichers MC, Barge-Schaapveld DQ, Nicolson NA, Peeters F, de Vries M, Mengelers R, et al. Reduced stress-sensitivity or increased reward experience: the psychological mechanism of response to antidepressant medication. Neuropsychopharmacology 2009; 34: 923-31.

29 Shrivastava A, Johnston M, Shah N, Bureau Y. Redefining outcome measures in schizophrenia: integrating social and clinical parameters. Curr Opin Psychiatry 2010; 23: 120-6.

30 Hofer A, Baumgartner S, Bodner T, Edlinger M, Hummer M, Kemmler G, et al. Patient outcomes in schizophrenia II: the impact of cognition. Eur Psychiatry 2005; 20: 395-402.

31 Harvey PD, Bellack AS. Toward a terminology for functional recovery in schizophrenia: is functional remission a viable concept? Schizophr Bull 2009; 35: 300-6.

32 Kane JM, Leucht S, Carpenter D, Docherty JP. The expert consensus guideline series. Optimizing pharmacologic treatment of psychotic disorders. Introduction: methods, commentary, and summary. J Clin Psychiatry 2003, 64 (suppl 12): 5-19.

33 Burns T, Patrick D. Social functioning as an outcome measure in schizophrenia studies. Acta Psychiatr Scand 2007; 116: 403-18.

34 Granholm E, Loh C, Swendsen J. Feasibility and validity of computerized ecological momentary assessment in schizophrenia. Schizophr Bull 2008; 34 507-14.

35 Myin-Germeys I, Birchwood M, Kwapil T. From environment to therapy in psychosis: a real-world momentary assessment approach. Schizophr Bull 2011; 37: 244-7

36 Helldin L, Kane JM, Karilampi U, Norlander T, Archer T. Remission in prognosis of functional outcome: a new dimension in the treatment of patients with psychotic disorders. Schizophr Res 2007; 93: 160-8.

37 Stone AA, Shiffman S, Schwartz JE, Broderick JE, Hufford MR. Patient compliance with paper and electronic diaries. Control Clin Trials 2003; 24 182-99.

38 Green AS, Rafaeli E, Bolger N, Shrout PE, Reis HT. Paper or plastic? Data equivalence in paper and electronic diaries. Psychol Methods 2006; 11: 87-105

39 Jacobs N, Nicolson NA, Derom C, Delespaul P, van Os J, Myin-Germeys I. Electronic monitoring of salivary cortisol sampling compliance in daily life. Life Sci 2005; 76: 2431-43.

40 Bellack AS. Scientific and consumer models of recovery in schizophrenia: concordance, contrasts, and implications. Schizophr Bull 2006; 32: 432-42. 